\title{
Bioassay Directed Identification of Royal Jelly's Active Compounds against the Growth of Bacteria Capable of Infecting Cutaneous Wounds
}

\author{
Mariana C. Garcia ${ }^{1}$, Mónica S. Finola ${ }^{1}$, Juan M. Marioli2 ${ }^{2 *}$ \\ ${ }^{1}$ Departamento de Microbiología e Inmunología, Universidad Nacional de Río Cuarto, Río Cuarto, Argentina \\ ${ }^{2}$ Departamento de Química, Universidad Nacional de Río Cuarto, Río Cuarto, Argentina \\ Email: juanmmarioli@gmail.com
}

Received January 17, 2013; revised February 17, 2013; accepted March 17, 2013

Copyright (C) 2013 Mariana C. Garcia et al. This is an open access article distributed under the Creative Commons Attribution License, which permits unrestricted use, distribution, and reproduction in any medium, provided the original work is properly cited.

\begin{abstract}
Antibiotic-resistant bacteria continue to be of major health concern world-wide. Thus, it is of great interest to study the biological properties and determine active compounds in natural products likely to be used as new health remedies. Therefore, the main objective of this work is to test the antibacterial activity of royal jelly samples, defatted royal jelly samples and their ethyl ether extracts against bacteria capable of infecting cutaneous wounds in humans and animals, and to recognize major bioactive compounds by using bioassay directed identification. The microorganisms used in the study were Staphylococcus aureus (including Methicillin-resistant and sensitive strains), Staphylococcus epidermidis, Micrococcus luteus, Streptococcus uberis, Enterococcus faecalis, Pseudomonas aeruginosa, Escherichia coli, and Klebsiella pneumoniae. The activity of royal jelly samples to inhibit bacterial growth was assessed by using well-difussion tests. Direct bioautography was used to identify bioactivity, and uv-visible spectroscopy and gas chromatography-mass spectrometry were used to identify bioactive compounds. Overall, royal jelly samples showed higher growth inhibition activity against Gram positive bacteria as compared to Gram negative bacteria. The growth of bacterial strains belonging to the Enterococcus and Streptococcus genders was less affected by the presence of royal jelly than bacterial strains of the Staphylococcus gender did. Compounds with antibacterial activity were found in the ethyl ether extract of royal jelly samples. 10-hydroxy-2-decenoic acid was the major component identified in the purified fraction obtained by bioassay guided fractionation of the ethyl ether extract. In conclusion, bioactivity of royal jelly samples is mainly due to their 10-hydroxy-2-decenoic acid content.
\end{abstract}

Keywords: Royal Jelly; Antibacterial Activity; 10-Hydroxy-2-decenoic Acid; Bioautography

\section{Introduction}

The emergence of new infectious diseases, the resurgence of several infections that appeared to have been controlled and the increase in bacterial resistance have created the necessity for studies directed towards the development of new antimicrobials [1]. Consequently, scientific efforts are being made to study and develop new compounds to be used beyond conventional antibiotic therapy. These comprise probiotic strains $[2,3]$, wastederived extracts from olive oil and wine production [4], peptide isolates [5], vegetal extracts [6,7], and natural foodstuffs such as kefir [8], honey [9,10], propolis [11] and royal jelly $[12,13]$. Despite drug discovery technology diversification and reduced funding for natural pro-

${ }^{*}$ Corresponding author. duct-based drug discovery, natural products from plants and other biological sources remain an undiminished source of new pharmaceuticals [14]. Thus, it is of great interest to study the biological properties of natural products or its components likely to be used as new health remedies.

Royal jelly (RJ) is a secretion produced by the hypopharyngeal and the mandibular glands in the head of the nurse bees. It has a thick, milky appearance, with a slightly acid, pungent odor and a somewhat bitter taste. The water content is fairly uniform, greater than $60 \%$, and with a water activity $\left(\mathrm{a}_{\mathrm{w}}\right)$ above 0.92 . The dry matter of RJ is composed of: protein $(27 \%-41 \%$, including free amino acids), carbohydrates (approximately $30 \%$ ), lipids $(8 \%-19 \%)$, trace elements and some vitamins [15]. RJ serves as nourishment for the honeybee larvae during 
their first three days of life and as the sole food for the queen bee during its entire life span.

Beyond its excellent nutritious properties, RJ has widely recognized biological actions, including vasodilative and hypotensive activities, disinfectant action, and antitumor, antihypercholesterolemic, and anti-inflammatory activities [16]. It was also reported that RJ has antibacterial activity against both Gram-positive and Gram-negative bacteria [17]. These authors attributed the antibacterial activity mainly to fatty acids present in RJ, such as trans10-hydroxydec-2-enoic acid, 3-hydroxydodecanoic acid, 11-oxododecanoic acid, and 11-S-hydroxydodecanoic acid. They also proposed that the short extension of the peptides, their poor cytolytic and mast cell degranulating activities, together with their broad spectrum antibiosis, make them attractive models for the development of new antibiotic agents.

Bacterial infections of the skin and underlying soft tissues are one of the most common presentations in patients visiting emergency room clinics in both hospitals and office based practices [18]. Microorganisms such as Staphylococcus aureus, Staphylococcus epidermidis, Micrococcus luteus, Streptococcus uberis, Enterococcus faecalis, Pseudomonas aeruginosa, Escherichia coli, and Klebsiella pneumoniae are frequently isolated from skin wounds in humans and animals. Methicillin-resistant and sensitive Staphylococcus aureus (MRSA and MSSA) are the main strains involved in difficult-to-treat skin and underlying tissue infections associated with gram-positive bacteria [19]. Staphylococcus epidermidis infections are commonly acquired in the hospital as a result of contamination of surgical cuts with microorganisms from the patients themselves or from the hospital personnel [20]. Infection with Pseudomonas aeruginosa is the most serious complication in burnt patients [21,22] followed by infections with Klebsiella pneumoniae, Escherichia coli, Staphylococcus aureus and other pathogen microorganisms [21]. Thus, we considered it necessary to undertake studies to determine the antibacterial activity of natural products against these significant microorganisms.

In this work, we identified the compound that makes royal jelly active against microorganisms capable of infecting cutaneous wounds. Entire RJ samples, as well as their defatted and lipidic extracts, were tested against Staphylococcus aureus, Staphylococcus epidermidis, Micrococcus luteus, Streptococcus uberis, Enterococcus faecalis, Pseudomonas aeruginosa, Escherichia coli, and Klebsiella pneumoniae. To the best of our knowledge, this is the first trial of RJ and its defatted and lipidic fractions against bacteria capable of infecting cutaneous wounds and demonstrates their high sensibility to RJ's components. A bioassay-directed fractionation of RJ's active compounds showed trans-10-hydroxy-2-decenoic acid as the most bioactive compound.

\section{Materials and Methods}

\subsection{Chemicals}

10-hydroxy-(E)2-decenoic (10-HDA) acid was purchased from Contech Inc., (Canada). 2,3,5-triphenyl tetrazolium chloride, dimethyl sulfoxide, bis(trimethylsilyl)trifluoroacetamide (BSTFA) and pyridine, were purchased from Sigma-Aldrich (Argentina). Thin layer chromatography plates (silica layer, 2 - $25 \mu \mathrm{m}$ diameter, $60 \AA$ pore size, $0.2 \mathrm{~mm}$ thick) and silica powder $(60 \AA, 35-75 \mu \mathrm{m})$ were from Fluka (USA).

\subsection{Gas Chromatographic Instrumentation}

An Agilent 6890 gas chromatograph equiped with a Flame Ionization Detector (GC-FID) and a Hewlett-Packard 5890 equiped with a mass spectrometric detector (GCMS) were used in the analysis of 10-HDA and the bioactive fractions. Gas chromatographic conditions were: column HP5 (30 m, $0.25 \mathrm{~mm}$ inner diameter, $0.25 \mu \mathrm{m}$ film thickness) and $250^{\circ} \mathrm{C}$ injector temperature. The temperature of the oven column was changed from $70^{\circ} \mathrm{C}$ to $250^{\circ} \mathrm{C}$ at $5^{\circ} \mathrm{C} / \mathrm{min}$. Nitrogen gas at $1 \mathrm{~mL} / \mathrm{min}$ was used as the carrier in the GC-FID analysis with a detector temperature of $250^{\circ} \mathrm{C}$. Helium gas at $1 \mathrm{~mL} / \mathrm{min}$ was used as the carrier in the GC-MS experiment and a detector temperature of $280^{\circ} \mathrm{C}$. The EIMS spectra were obtained at 70 $\mathrm{eV}$ of ionization energy. Detection was performed in full scan mode from 40 to 620 a.m.u.

\subsection{Royal Jelly Samples}

Royal jelly samples were obtained from four sources: one sample produced in southern Córdoba (Argentina) was acquired in the local market (named RJ R), another sample was provided by Estación Experimental Agropecuaria INTA Famaillá, Tucumán (Argentina) from an organic farm (named RJ NG), another one was provided by a producer from La Pampa province, Argentina (named RJ LP) and the fourth sample was kindly provided by producers from the city of Corrilobo, Córdoba (Argentina) (named sample RJ C). Royal jelly samples were stored in sterile plastic flasks in a cold $\left(4^{\circ} \mathrm{C}-8^{\circ} \mathrm{C}\right)$ and dark place. The samples fulfill the requirements of the Código Alimentario Argentino [23]. For the agar-well diffusion assays, royal jelly samples were used, without centrifugation, undiluted and at $90 \%, 80 \%, 70 \%, 60 \%$, $50 \%, 40 \%, 30 \%, 20 \%$ and $10 \%$ dilutions (grams of royal jelly diluted with water to a final weight of $100 \mathrm{~g}$ ).

\subsection{Royal Jelly Fractionation}

Lyophilized royal jelly samples were fractioned into ethyl ether-soluble compounds and defatted-royal jelly samples by using a Soxhlet extractor. Ethyl ether was then evaporated under partial vacuum in a rotary evaporator 
$\left(30^{\circ} \mathrm{C}\right)$ leaving an oily residue. Defatted royal jelly samples were freed from ethyl ether under partial vacuum in a vacuum oven $\left(30^{\circ} \mathrm{C}\right)$.

Ethyl ether soluble compounds were further separated by liquid-solid chromatography on silica gel using ethyl ether-hexane $(65: 35 \mathrm{v} / \mathrm{v})$ as the mobile phase. Fractions of approximately $2 \mathrm{~mL}$ were collected in glass vials. The content of each glass vial was analyzed by thin layer chromatography (TLC) and those vials showing a TLC band with $\mathrm{Rf}=0.27$ were collected all together, the solvent was evaporated under partial vacuum, and the dried material kept in a dark vial at $4^{\circ} \mathrm{C}-8^{\circ} \mathrm{C}$ until use.

\subsection{Sample Preparation for GC Analysis}

Approximately $0.5 \mathrm{~mL}$ of the 10-HDA standard or the bioactive band (purified as described in the previous section) was put into a vial of $2 \mathrm{~mL}$. Then, $220 \mathrm{~mL}$ of pyridine and $80 \mathrm{~mL}$ of BSTFA were added. The reaction mixture was sealed and heated at $60^{\circ} \mathrm{C}$ during $30 \mathrm{~min}$ to obtain the trimethylsilyl (TMS) derivatives.

\subsection{Bacterial Strains}

Reference strains of E. faecalis 1 (ATCC 29212), $S$. aureus MS 1 (ATCC 25923), S. agalactiae 1 (ATCC 27956), S. dysgalactiae 2 (ATCC 27957), and M. luteus (ATCC 9341) were used. Strain of S. epidermidis was isolated from cow milk. Strains of S. uberis, S. agalactiae 2 and 3, and of $S$. dysgalactiae 2 and 3, were isolated from cow mastitis. Strains of E. coli and of $P$. aeruginosa 1 were isolated from well water; and $K$. pneumoniae isolated from poultry manure were also used in this work. These strains were kindly donated by the Microbiology Laboratory of Universidad Nacional de Río Cuarto. Strains of $S$. aureus MS 2, S. aureus MR 1 and $S$. aureus MR 2, isolated from milk obtained from cows with mastitis, were kindly donated by the Bacteriology Laboratory of Universidad Federal Rural de Rio de Janeiro. Strains of $E$. faecalis 2, 3 and 4, and of E. faecium were isolated from skin infections in humans. The strain of $P$. aeruginosa 2 was isolated from a catheter infection. All bacterial cultures but those of the Streptococcus sp. were kept in brain-heart infusion with $15 \%$ glycerol and maintained in $3 \mathrm{~mL}$ plastic bottles at $-20^{\circ}$ C. Streptococcus sp. strains were kept in skim milk yeast extract with $10 \%$ glycerol and maintained in $3 \mathrm{~mL}$ plastic bottles at $-20^{\circ} \mathrm{C}$. All the bacterial cultures were diluted in trypticase soy broth (TSB) until an Optical Density (O.D.) in the range of $0.02-0.04$ was obtained, which corresponded to a colony count between $1 \times 10^{6}$ and $1 \times 10^{7} \mathrm{CFU} / \mathrm{mL}$.

\subsection{Growth Inhibition Analysis: Agar-Well Diffusion Assays}

Agar-well diffusion assays were carried out with each of the above-mentioned bacterial strains. Two hundred microliters of the bacterial culture were poured onto the Petri dish containing Mueller Hinton Agar (MHA) and spread with a Drigalsky spatula until the microorganisms were absorbed. Holes of about $5 \mathrm{~mm}$ diameter were drilled on the culture medium and $50 \mu \mathrm{L}$ of each royal jelly dilution were added to each hole. Sterilized water was used as negative control. Plates were incubated at $37^{\circ} \mathrm{C}$ for $24 \mathrm{~h}$. The diameters of the circular inhibition zones obtained were measured with a caliper. The experiments were repeated three times. Minimum inhibittory concentrations (MICs) were determined as the last royal jelly dilution which gives a measurable inhibition zone.

Stock solutions of the isolated bioactive band (24 $\mathrm{mg} / \mathrm{mL})$ and of $10-\mathrm{HDA}(26 \mathrm{mg} / \mathrm{mL})$ were prepared in 50:50 DMSO-0.15\% agar-agar for the study of their growth inhibition activity.

\subsection{Statistical Analysis}

Bacterial strains were grouped into four categories for the statistical analysis: Enterococcus sp., Staphylococcus sp., Streptococcus sp., and Gram negative bacteria. MIC values for each group were computed as the average of individual MIC values of each bacterial strains in the group. Statistical software R version 2.12.2 [24] was used for the two-tailed ANOVA computations.

\section{Results}

Table 1 shows the results of the antibacterial activity

Table 1. MIC data (percent w/w.) of royal jelly samples against some bacteria capable of infecting cutaneous wounds in humans and animals.

\begin{tabular}{lcccc}
\hline Bacterial strain & RJ R & RJ NG & RJ C & RJ LP \\
\hline E. faecalis 1 & 80 & 80 & 60 & 80 \\
E. faecalis 2 & 80 & 90 & 40 & 100 \\
E. faecalis 3 & N. I. & 70 & 40 & 80 \\
E. faecalis 4 & 90 & 80 & 60 & 50 \\
E. faecium & N. I. & 70 & 50 & 50 \\
S. aureus MS 1 & 60 & 20 & 80 & 50 \\
S. aureus MS 2 & 60 & 50 & 40 & 80 \\
S. aureus MR 1 & 70 & 40 & 60 & 70 \\
S. aureus MR 2 & 70 & 60 & 30 & 60 \\
S. epidermidis & 80 & 40 & 50 & 50 \\
S. agalactiae 1 & 90 & 80 & 50 & N. I. \\
S. agalactiae 2 & 90 & 50 & 80 & 100 \\
S. agalactiae 3 & 90 & 90 & 90 & 70 \\
S. dysgalactiae 1 & 100 & 60 & 50 & 90 \\
S. dysgalactiae 2 & 80 & 100 & 90 & N. I. \\
S. dysgalactiae 3 & 80 & 80 & 90 & N. I. \\
S. uberis & N. I. & 70 & 60 & N. I. \\
M. luteus & 40 & 60 & 50 & 60 \\
E. coli & 100 & 60 & 80 & 70 \\
K. pneumoniae & N. I. & N. I. & 100 & 80 \\
P aeruginosa 1 & 100 & N. I. & 80 & 70 \\
P. aeruginosa 2 & 90 & 100 & 80 & 60 \\
\hline in
\end{tabular}

*indicates a reference strain; N. I. indicates no inhibition. 
studies of royal Jelly samples from different geographic provenance against various bacterial strains capable of infecting cutaneous wounds in humans and animals. The minimum inhibitory concentration of royal jelly samples (grams of royal jelly per $100 \mathrm{~g}$ solution) is tabulated for each of the bacterial strain tested in this study.

The average MIC data obtained for each bacterial group and for each Royal Jelly sample geographic provenance were compared by two-way ANOVA. This measured the differences between the bacterial groups, the geographic provenance of the RJ samples, and their interaction. No significant interaction between bacterial group and RJ sample geographic provenance was observed $(p>0.05)$. On the other side, significant differences $(p<0.05)$ were observed in the average MIC data of the factors bacterial groups and those of royal jelly sample geographic provenance. The test of Tukey was then performed to compare average MIC data between the bacterial groups and between RJ sample geographic provenances. Table 2 shows the average MIC data and standard deviations obtained in the growth inhibition tests with the different bacterial groups. Table 3 shows the results obtained (average MIC data and standard deviations) in the growth inhibition tests using RJ samples of different geographic provenance.

Fractionation of royal jelly samples into defatted extracts and ethyl ether-soluble substances allowed determining which one carried the bioactive compounds. Ethyl ether-soluble compounds were always more active in inhibiting bacterial growth than defatted extracts. Thus, we continued with the analysis of the ethyl ether-soluble substances to determine the identity of bioactive compounds.

Ethyl ether-soluble compounds were separated by thin layer chromatography. Five well-defined chromatographic bands were observed in the TLC analysis of the ethyl ether-soluble fraction of the different RJ samples using a mixture of ethyl ether:hexane $(65: 35 \mathrm{v} / \mathrm{v})$. The average $\mathrm{Rf}$ values of the TLC bands were: $0.98,0.57,0.27,0.18$ and 0.07 .

Direct bioautography, used for qualitative antibacterial activity detection, indicated that the ethyl ether-soluble extract has a band $(\mathrm{Rf}=0.27)$ with compounds showing high antibacterial activity against $S$. aureus MS 1. Thus, the ether-soluble extract was submitted to flash chromatography to isolate the more active compounds.

The isolated bioactive band was then analyzed by uv-visible spectroscopy, GC-FID and GC-MS. The uvvisible spectrum of the isolated bioactive band in methanol showed an absorbance maximum at $215 \mathrm{~nm}$. A matching spectrum was obtained with a standard of 10-hydroxy 2-decenoic acid. The GC-FID analysis of the isolated bioactive compound showed a major peak at $12.4 \mathrm{~min}$ and a minor peak at $11.4 \mathrm{~min}$ (Figure 1). The GC-FID analysis of the 10-HDA standard showed a peak at 12.4 min (inset in Figure 1). These results are indicative of the identity of the component with a retention time of $12.4 \mathrm{~min}$. Gas chromatography with mass spectrometric detection can identify the component of a chromatographic peak. Matching MS spectra of the major chromatographic peaks were found in the GC-MS analysis of both the isolated bioactive band and the 10-HDA standard. The following MS data (target ion $\mathrm{m} / \mathrm{z}$ (Signal intensity)) 147(100), 315(96), 73(64), 75(43), 81(37) was obtained in the MS analysis of the standard. The mass spectra of the main peak observed in the GC-MS analysis of the bioactive band agreed with that of the standard, when considering normal variability. Thus, we concluded that the main component of the bioactive band is 10-HDA.

Finally, the antibacterial activities of the isolated bioactive band and of 10-HDA were assessed. Table 4 shows the results (average MIC data \pm standard deviation) obtained in the growth inhibition tests analysis.

\section{Discussion}

It can be seen at first glance in Table 1 that the growth of all the tested bacterial strains was inhibited by some of the royal jelly samples studied. Overall, the royal jelly sample from Corrilobo (RJ C) seemed the most active given that it inhibited all the bacterial strains tested. The royal jelly sample from Norte Grande (RJ NG) inhibited $91 \%$ of the bacterial strains tested while Regional royal jelly and that from La Pampa (RJ R and RJ LP) seemed less active given that they inhibited $82 \%$ of the bacterial strains tested.

Table 2. Mean \pm SD (\%w/w) of MIC data obtained in the growth inhibition tests for the different bacterial groups.

\begin{tabular}{cccc}
\hline & Staphylococcus sp. & Enterococcus sp. & Streptococcus sp. \\
\hline MIC & $53.3 \pm 16.5^{\mathrm{a}}$ & $69.5 \pm 18.0^{\mathrm{b}}$ & $74.5 \pm 16.9^{\mathrm{b}}$ \\
\hline
\end{tabular}

Different superscript letters indicate statistically significant differences $(\mathrm{p}<0.05)$ between bacterial groups MIC.

Table 3. Mean \pm SD $(\% \mathrm{w} / \mathrm{w})$ of MIC data obtained in the growth inhibition tests using royal jelly samples of different provenance.

\begin{tabular}{cccc}
\hline & Corrilobo & Norte Grande & La Pampa \\
\hline MIC & $59.8 \pm 17.7^{\mathrm{a}}$ & $67.9 \pm 21.5^{\mathrm{a}, \mathrm{b}}$ & $71.2 \pm 16.5^{\mathrm{b}, \mathrm{c}}$ \\
\hline
\end{tabular}

Different superscript letters indicate statistically significant differences $(\mathrm{p}<0.05)$ between bacterial groups MIC. 


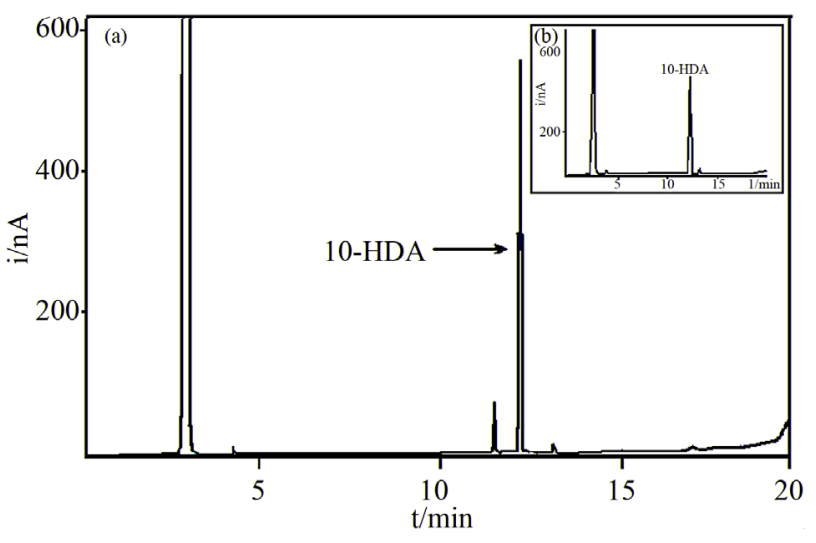

Figure 1. Gas chromatograms with Flame Ionization Detection of: (a) The bioactive band; (b) A standard of 10-HDA. Gas chromatographic conditions are given in the "Methods and Materials" section.

Table 4. MIC data (average $\mathrm{mg} / \mathrm{mL} \pm$ SD) of the isolated bioactive band and of 10-HDA against some bacteria capable of infecting cutaneous wounds in humans and animals.

\begin{tabular}{lcc}
\hline \multicolumn{1}{c}{ Bacterial strain } & Isolated bioactive band & 10-HDA \\
\hline E. faecalis 1 & $2.0 \pm 0.7$ & $2.3 \pm 0.8$ \\
E. faecalis 2 & $1.6 \pm 0.7$ & $1.9 \pm 0.8$ \\
E. faecalis 3 & $1.6 \pm 0.7$ & $1.9 \pm 0.8$ \\
E. faecalis 4 & $2.0 \pm 0.7$ & $2.3 \pm 0.8$ \\
E. faecium & $2.0 \pm 0.7$ & $2.3 \pm 0.8$ \\
S. aureus MS 1 & $1.6 \pm 0.7$ & $1.9 \pm 0.8$ \\
S. aureus MS 2 & $1.6 \pm 0.7$ & $1.9 \pm 0.8$ \\
S. aureus MR 1 & $1.6 \pm 0.7$ & $1.9 \pm 0.8$ \\
S. aureus MR 2 & $2.0 \pm 0.7$ & $2.3 \pm 0.8$ \\
S. agalactiae 1 & N. I. & N. I. \\
S. agalactiae 2 & $2.0 \pm 0.7$ & $2.3 \pm 0.8$ \\
S. agalactiae 3 & $0.8 \pm 0.4$ & $0.9 \pm 0.4$ \\
S. uberis & $1.6 \pm 0.7$ & $2.3 \pm 0.8$ \\
E. coli & N. I. & N. I. \\
K. pneumoniae & N. I. & N. I. \\
P. aeruginosa 2 & N. I. & N. I. \\
\hline
\end{tabular}

indicates a reference strain.

The MIC value for the various strains tested in this study may be considered indicative of the RJ antibacterial activity. As regards RJ R, high antibacterial activity was observed for Gram positive strains as can be deduced from their MIC values (e.g. $40 \%$ for M. luteus). Similar results of antibacterial activity of RJ against $M$. luteus were informed in the literature by other authors [25]. Gram negative strains were less sensitive to RJ R given that some strains were not inhibited at all (e.g. $K$. pneumoniae), only the undiluted sample inhibited E. coli and $P$. aeruginose 1 , and $90 \% \mathrm{RJ} \mathrm{R}$ inhibited $P$. aeruginose 2 . These findings are in agreement with those in- formed in the literature by other authors [26,27].

Regarding RJ NG, a noticeable antibacterial activity was observed against most of the bacterial strains tested in this study. Only the growth of K. pneumonia and of $P$. aeruginosa 1 was not inhibited by RJ NG. As with RJ R, Gram positive bacteria seemed more sensitive to RJ NG than Gram negative bacterial strains did. From the latter group, E. coli was the most sensitive with a MIC value of $60 \%$ and $K$. pneumoniae was the most resistant considering that RJ NG did not inhibit its growth. On the other side, $S$. aureus MS 1 showed the highest sensitivity to RJ NG of all the bacterial strain tested in this study, with a MIC value of $20 \%$.

$\mathrm{RJ} C$ also produced a noticeable inhibition of bacterial growth. E. faecalis 2 and 3 were the most sensitive bacterial strains of the Enterococcus genus, both with a MIC value of $40 \%$. It is worth noting the MIC value of the $S$. aureus $\mathrm{MR} 2$ bacterial strain (MIC $=30 \%$ ), which may be interesting given that methicillin resistant bacteria can cause serious infections of cutaneous wounds in humans and animals. Methicillin resistant bacteria may also be resistant to other $\beta$-lactam antibiotics, to chloramphenicol, to tetracyclines, to macrolides, to aminoglucosides and to quinolones inclusive. RJ C seemed the most active $\mathrm{RJ}$ sample considering that it inhibited all the bacterial strains tested in this study.

Finally, RJ LP also showed a noticeable inhibition of the growth of different bacterial strains, Gram positive bacteria seemed more sensitive than Gram negative bacteria. Bacterial strains isolated from skin wounds showed a marked sensitivity to the presence of RJ LP. Thus, $E$. faecalis 1 and E. faecium were the most sensitive bacterial strains given that a concentration of $50 \% \mathrm{RJ}$ LP was enough to inhibit bacterial growth. However, E. faecalis 2 seemed the most resistant bacteria strain because it was only inhibited by pure RJ LP.

It can be seen in Table 2 that the Staphylococcus sp. group was the most sensitive group to RJ samples of any geographic provenance, while no significant differences were observed among Enterococcus sp., Streptococcus sp. and Gram negative bacterial groups. On the other hand, Corrilobo and Norte Grande RJ samples were the most active against the growth of any bacterial group considering that their average MIC data were smaller than those obtained with RJ samples from La Pampa and Regional (Table 3). In a study of the substances present in RJ samples from different geographic provenance in Turkey, Stocker [25] showed that the geographic provenance of the RJ sample influenced its antibacterial activeity. Similar results were found in our study pointing to the significance of the RJ geographic provenance on its biological activity.

Lercker et al. [28] identified the organic acid families present in royal jelly samples. By using TLC and gas 
chromatography-mass spectrometry they showed that the lipid fraction of royal jelly is mainly composed of polar substances and that non-polar compounds are only a small fraction. Moreover, they showed that mono- and di-carboxylic fatty acids, sterols, and mono- and di-hydroxyl fatty acids are the main components of the polar substances.

It can be observed in Table 4 that both the isolated bioactive band and the 10-HDA standard showed the highest antibacterial activity against $S$. agalactiae 3 . Conversely, S. agalactiae $1, E$. coli, $K$. pneumonia and $P$. aeruginosa 2 were the most resistant bacterial strains considering that neither the isolated active band nor the 10-HDA standard inhibited their growth. On the other hand, close similarities of MIC values were observed between the isolated bioactive band and the 10-HDA standard when compared on the same bacterial strain. These results may also indicate that the isolated bioactive band is mainly composed of 10-hydroxy 2-decenoic acid, and that this is the compound with the highest bioactivity in royal jelly.

\section{Conclusion}

The growth of strains of Staphylococcus aureus (including Methicillin-resistant and sensitive strains), Staphylococcus epidermidis, Micrococcus luteus, Streptococcus uberis, Enterococcus faecalis, Pseudomonas aeruginosa, Escherichia coli, and Klebsiella pneumoniae was inhibited by royal jelly or by its ethyl ether extract. This finding is important because these bacteria are capable of infecting cutaneous wounds in humans and animals, causing major health treatment problems in humans or several economic loss when milk cows are infected. High antibacterial activity with minimum inhibitory concentrations values as low as $30 \%(\mathrm{w} / \mathrm{w})$ were observed on Gram positive bacteria when entire royal jelly samples were used. Less bioactivity was observed on Gram negative bacteria. The ethyl ether-soluble fraction of royal jelly showed higher bioactivity for the inhibition of bacterial growth as compared with defatted royal jelly samples. MIC values for the ethyl ether-soluble fractions were in the range from 0.6 to $2.4 \mathrm{mg} / \mathrm{mL}$. Thin layer chromatography and bioautography-guided fractionation of the ethyl ether-soluble compunds allowed us to identify the most bioactive chromatographic band, and GCFID and GC-MS experiments showed that 10-hydroxy2 -decenoic acid was the main component of this bioactive chromatographic band.

\section{Acknowledgements}

This work was supported by grants from Secretaría de Ciencia y Técnica of Universidad Nacional de Río Cuarto and Agencia Nacional de Promoción Científica y
Tecnológica. We are grateful to Prof. Mery Picco statistical analysis help. M. C. G. thanks CONICET (Argentina) for her research fellowship. J. M. M. is an Independent Researcher of CONICET.

\section{REFERENCES}

[1] C. Valgas, S. Machado de Souza, E. F. A. Smânia and A. Smânia Jr., "Screening Methods to Determine Antibacterial Activity of Natural Products," Brazilian Journal of Microbiology, Vol. 38, No. 2, 2007, pp. 369-380. doi:10.1590/S1517-83822007000200034

[2] G. Reid, "Probiotic Agents to Protect the Urogenital Tract against Infection," American Journal of Clinical Nutrition, Vol. 73, No. 2, 2001, pp. 437S-443S.

[3] S. Petti, G. Tarsitani and A. Simonetti D'Arca, “Antibacterial Activity of Yoghurt against Viridans Streptococci in Vitro," Archives of Oral Biology, Vol. 53, No. 10, 2008, pp. 985-990. doi:10.1016/j.archoralbio.2008.04.009

[4] A. T. Serra, A. A. Matias, A. V. M. Nunes, M. C. Leitão, D. Brito, R. Bronze, et al., "In Vitro Evaluation of Oliveand Grape-Based Natural Extracts as Potential Preservatives for Food," Innovative Food Science and Emerging Technologies, Vol. 9, No. 3, 2008, pp. 311-319. doi:10.1016/j.ifset.2007.07.011

[5] Y. H. Yau, B. Ho, N. S. Tan, M. L. Ng and J. L. Ding, "High Therapeutic Index of Factor C Sushi Peptides: Potent Antimicrobials against Pseudomonas aeruginosa," Antimicrobial Agents and Chemotherapy, Vol. 45, No. 10, 2001, pp. 2820-2825. doi:10.1128/AAC.45.10.2820-2825.2001

[6] M. T. Chomnawang, S. Surassmo, K. Wongsariya and N. Bunyapraphatsara, "Antibacterial Activity of Thai Medicinal Plants against Methicillin-Resistant Staphylococcus aureus," Fitoterapia, Vol. 80, No. 2, 2009, pp. 102104. doi:10.1016/j.fitote.2008.10.007

[7] M. J. González and J. M. Marioli, “Antibacterial Activity of Water Extracts and Essential Oils of Various Aromatic Plants against Paenibacillus larvae, the Causative Agent of American Foulbrood," Journal of Invertebrate Pathology, Vol. 104, No. 3, 2010, pp. 209-213. doi:10.1016/j.jip.2010.04.005

[8] K. Leite Rodrigues, L. R. Gaudino Caputo, J. C. Tavares Carvalho, J. Evangelista and J. M. Schneedorf, "Antimicrobial and Healing Activity of Kefir and Kefiran Extract," International Journal of Antimicrobial Agents, Vol. 25, No. 5, 2005, pp. 404-408.

[9] P. Molan, "Not All Honeys Are the Same for Wound Healing," European Tissue Repair Society Bulletin, Vol. 9, No. 1, 2002, pp. 5-6.

[10] C. Basualdo, V. Sgroy, M. S. Finola and J. M. Marioli, "Comparison of the Antibacterial Activity of Honey from Different Provenance against Bacteria Usually Isolated from Skin Wounds," Veterinary Microbiology, Vol. 124, No. 3-4, 2007, pp. 375-381. doi:10.1016/j.vetmic.2007.04.039

[11] A. C. H. F. Sawaya, K. S. Souza, M. C. Marcucci, I. B. S. Cunha and M. T. Shimizu, "Analysis of the Composition of Brazilian Propolis Extracts by Chromatography and 
Evaluation of Their in Vitro Activity against Gram-Positive Bacteria," Brazilian Journal of Microbiology, Vol. 35, No. 1-2, 2004, pp. 104-109. doi:10.1590/S1517-83822004000100017

[12] S. Eshraghi, "An Evaluation of the Potent Inhibitory Effects of Royal Jelly Fractions against Streptomyces Bacteria," Pakistan Journal of Medical Sciences, Vol. 21, No. 1, 2005, pp. 63-68.

[13] M. C. Garcia, M. S. Finola and J. M. Marioli, "Antibacterial Activity of Royal Jelly against Bacteria Capable of Infecting Cutaneous Wounds," Journal of ApiProducts and ApiMedical Science, Vol. 2, No. 3, 2010, pp. 93-99. doi:10.3896/IBRA.4.02.3.02

[14] B. Schmidt, D. M. Ribnicky, A. Poulev, S. Logendra, W. T. Cefalu and I. Raskin, "A Natural History of Botanical Therapeutics," Metabolism Clinical and Experimental, Vol. 57, No. 1, 2008, pp. S3-S9. doi:10.1016/i.metabol.2008.03.001

[15] A. G. Sabatini, G. L. Marcazzan, M. F. Caboni, S. Bogdanov and L. B. de Almeida-Muradian, "Quality and Standarisation of Royal Jelly," Journal of ApiProducts and ApiMedical Science, Vol. 1, No. 1, 2009, pp. 16-21. doi:10.3896/IBRA.4.01.1.04

[16] T. Nagai and R. Inoue, "Preparation and the Functional Properties of Water Extract and Alkaline Extract of Royal Jelly," Food Chemistry, Vol. 84, No. 2, 2004, pp. 181186. doi:10.1016/S0308-8146(03)00198-5

[17] E. Melliou and I. Chinou, "Chemistry and Bioactivity of Royal Jelly from Greece," Journal of Agricultural and Food Chemistry, Vol. 53, No. 23, 2005, pp. 8987-8992. doi:10.1021/jf051550p

[18] M. E. Jones, J. A. Karlowsky, D. C. Draghi, C. Thornsberry, D. F. Sahm and D. Nathwani, "Epidemiology and Antibiotic Susceptibility of Bacteria Causing Skin and Soft Tissue Infections in the USA and Europe: A Guide to Appropriate Antimicrobial Therapy," International Journal of Antimicrobial Agents, Vol. 22, No. 4, 2003, pp. 406419. doi:10.1016/S0924-8579(03)00154-7

[19] L. Halcón and K. Milkus, "Staphylococcus aureus and Wounds: A Review of Tea Tree Oil as a Promising An- timicrobial," American Journal of Infection Control, Vol. 32, No. 7, 2004, pp. 402-408.

doi:10.1016/j.ajic.2003.12.008

[20] C. Vuong and M. Otto, "Staphylococcus Epidermidis Infections," Microbes and Infection, Vol. 4, No. 4, 2002, pp. 481-489. doi:10.1016/S1286-4579(02)01563-0

[21] S. Nasser, A. Mabrouk and A. Maher, "Colonization of Burn Wounds in Ain Shams University Burn Unit," Burns, Vol. 29, No. 3, 2003, pp. 229-233. doi:10.1016/S0305-4179(02)00285-1

[22] U. Altoparlak, F. Aktas, D. Celebi, Z. Ozkurt and M. N. Akcay, "Prevalence of Metallo- $\beta$-Lactamase among Pseudomonas aeruginosa and Actinobacter baumanii Isolated from Burn Wounds and in Vitro Activities of Antibiotic Combinations against These Isolates," Burns, Vol. 31, No. 6, 2005, pp. 707-710. doi:10.1016/j.burns.2005.02.017

[23] A. A. Código, X. Capítulo and A. Alimentos, "Art. 784," 2010.

http://www.anmat.gov.ar/alimentos/normativas_alimentos caa.asp

[24] R Development Core Team. R: A language and environment for statistical computing. R Foundation for Statistical Computing, Vienna. 2010. http://www.R-project.org

[25] A. Stocker, "Isolation and Characterization of Substances from Royal Jelly," 2003.

[26] C. S. McCleskey and R. M. Melampy, "Bactericidal Properties of Royal Jelly of Honeybee," Journal of Economic Entomology, Vol. 32, No. 4, 1939, pp. 581-587.

[27] N. Sauerwald, J. Polster, E. Bengsch, L. Niessen and R. Vogel, "Combined Antibacterial and Antifungal Properties of Water Soluble Fractions of Royal Jelly," Advances in Food Science, Vol. 20, No. 1-2, 1998, pp. 46-52.

[28] G. Lercker, P. Capella, L. S. Conte, F. Ruini and G. Giordani, "Components of Royal Jelly: I. Identification of Organic Acids," Lipids, Vol. 16, No. 12, 1981, pp. 912-919. doi:10.1007/BF02534997 